\title{
Nonketotic hyperglycemia: The culprit behind refractory seizures in a case of traumatic brain injury
}

Sir,

Seizure following Traumatic Brain Injury is a routinely encountered situation, but those induced by hyperglycemic state is rather uncommon. Such a scenario of hyperglycemic seizures in the setting of traumatic brain injury may often go unrecognized.

The patient being reported is a 54 year old male who met with a road traffic accident while he was travelling in his motorcycle without a helmet. He had sustained injury to the right side of his head and a few abrasions over his arms and legs. He did not have any episode of loss of consciousness or seizure. He was brought to our hospital in less than an hour. His plain CT brain showed a focal parenchymal haemorrhage with mild surrounding oedema in the right posterior temporal region. A thin rim of extra-axial haemorrhage was seen at the right basal temporal fossa, along with subarachnoid haemorrhage in bilateral temporal and occipital sulci. There was no midline shift (Figure 1). He was not a known diabetic or hypertensive; and was not on any regular medications. He was conscious and oriented. His vitals and systemic examinations were normal. His blood investigations like complete blood count, renal and liver functions, electrolytes, ECG and chest Xray were

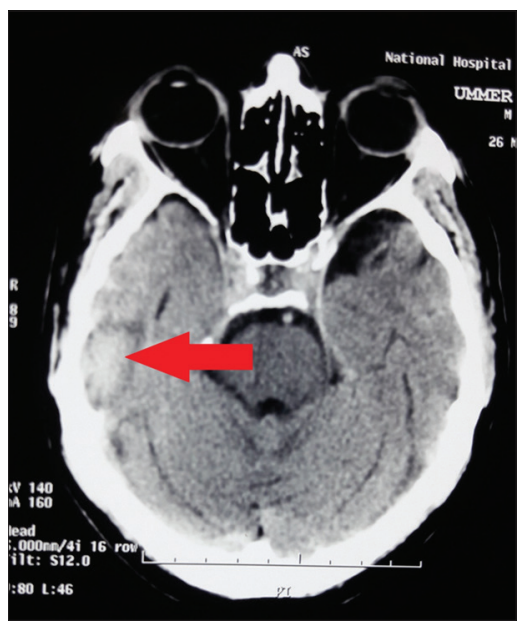

Figure 1: CT brain showing focal parenchymal haemorrhage in the right posterior temporal region normal. His random blood sugar was $117 \mathrm{mg}^{\%}$ at the time of admission. Neurosurgery opinion was taken and medical management was proposed. Intravenous 20\% Mannitol was started. The patient continued to be asymptomatic. The following day, he started developing focal seizures starting from his left upper limb with secondary generalization lasting about 3 minutes; with a post ictal state lasting about 15 minutes. He was given lorazepam and loading dose phenytoin intravenously. However, he continued to have similar seizure episodes almost every 30 minutes. His repeat CT brain did not show any fresh changes. At this point, his random blood sugars were $627 \mathrm{mg}^{0} \%$ and HbA1c was $9.8 \%$, with normal electrolytes. His urine ketones were negative, serum osmolality $344 \mathrm{mOsm} / \mathrm{mL}$ and arterial pH 7.32. Mannitol was withheld and he was managed with intravenous fluids and insulin infusion (as per treatment guidelines for hyperglycemic hyperosmolar state). Maintenance dose of intravenous phenytoin was continued. Following the normalization of his glucose levels, there were no further episodes of seizure. His EEG showed mild electrophysiological dysfunction on the right side. He was discharged after 5 days of hospital stay on insulin alone, without any antiepileptic drugs. He has remained seizure free over the next 3 months of his follow-up.

Seizures following traumatic brain injury may be partial, with or without alteration of consciousness; or with secondary generalization. The temporal lobe is one of the most common sites for post-traumatic seizures. ${ }^{1}$ Most cases of temporal lobe seizures are associated with an aura, which may be psychic (fear, anxiety, déjà vu) or autonomic (abdominal discomfort, nausea) or olfactory and gustatory hallucinations. This may be followed by altered sensorium or staring look with responsiveness. However, secondary generalizations are uncommon with seizures of temporal lobe. ${ }^{2}$ 
Seizures associated with hyperglycemia are usually partial type. The first case of hyperglycemic focal seizures was reported in $1965 .{ }^{3}$ These are commonly seen with nonketotic hypergycemia and are refractory to antiepileptic drugs. They resolve with insulin therapy and hydration. The mechanism of seizures in hyperglycemia remains unclear. One hypothesis suggests a decrease in the seizure threshold due to metabolic disturbance. ${ }^{4}$ The inhibition of Krebs cycle, leading to increased metabolism of gamma amino-butyric acid can also lower the threshold for seizure activity. ${ }^{5}$ Focal seizures can also be triggered by hyperosmolality and hyperglycemia induced dehydration. Hyperglycemia can cause reversible focal ischemia without any structural damage by decreasing blood flow in certain areas of the brain. ${ }^{6}$

Nonketotic hyperglycemic seizures are rare and refractory to anticonvulsant drugs. In this case, the patient had a traumatic temporal lobe haemorrhage; and in such a scenario, hyperglycemic seizures may get misdiagnosed, especially if the patient is not a known diabetic. The nonresolution of seizures with antiepileptic drugs should strongly raise the suspicion of hyperglycemia as one of the culprits.

\section{Robin George Manappallil', Ramesh Naga Supreeth ${ }^{2}$}

${ }^{1}$ Consultant-Physician, ${ }^{2}$ Resident, Department of Internal Medicine, Baby Memorial Hospital, Calicut, Kerala, India
Address for correspondence: Dr. Robin George Manappallil, Consultant - Physician, Baby Memorial Hospital, Calicut, Kerala 673004, India. E-mail: drrobingeorgempl@gmail.com Phone: 0091-8547753396

Key words: Seizure, Hyperglycemia, Temporal lobe epilepsy, Traumatic brain injury

\section{REFERENCES}

1. Gupta PK, Sayed N, Ding K, Agostini MA, Van Ness PC, Yablonet S, et al. Subtypes of Post-Traumatic Epilepsy: Clinical, Electrophysiological, and Imaging Features. Journal of Neurotrauma 2014; 31(16):1439-1443.

2. Ding K, Gupta PK and Diaz-Arrastia R. Epilepsy after Traumatic Brain Injury. In: Laskowitz D, Grant G, editors. Translational Research in Traumatic Brain Injury. Boca Raton (FL): CRC Press/Taylor and Francis Group; 2016. Chapter 14.

3. Maccario M, Messis CP and Vastola F. Focal seizures as a manifestation of hyperglycemia without ketoacidosis. Neurology 1965;15:195-206.

4. Singh BM, Gupta DR and Strobos RJ. Nonketotic hyperglycemia and epilepsia partialis continua. Arch Neurol 1973;29(3):187-190.

5. Brick JF, Gutrecht JA and Ringel RA. Reflex epilepsy and nonketotic hyperglycemia in the elderly: A specific neuroendocrine syndrome. Neurology 1989;39(3):394-399.

6. Duckrow RB, Beard DC and Brennan RW. Regional cerebral blood flow decreases during hyperglycemia. Ann Neurol 1985; 17(3):267-272.

\footnotetext{
Authors Contribution:

RGM - Concept and design of case report, reviewed the literature, manuscript preparation, critical revision of manuscript and treating physician;

RNS - Manuscript preparation and resident incharge.
}

Source of Support: Nil, Conflict of Interest: None declared. 